\title{
A study of coronary artery patency in relation to the index event in patients with myocardial infarction thrombolysed with streptokinase
}

\author{
R K Gokhroo, Sajal Gupta, Devendra Singh Bisht, Deepak Padmanabhan
}

Department of Cardiology, Institute of Cardiology, JLN Medical College and Associated Group of Hospitals, Ajmer, Rajasthan, India

\section{Correspondence to} Dr Sajal Gupta, Department of Cardiology, Institute of Cardiology, JLN Medical College and Associated Group of Hospitals, 22/16, Vaishali Nagar, Ajmer, Rajasthan 305 001, India; drsajal@yahoo.com

Received 8 January 2014 Revised 22 March 2014 Accepted 28 March 2014
CrossMark

\footnotetext{
To cite: Gokhroo RK, Gupta S, Bisht DS, et al. Heart Asia 2014;6:55-58. doi:10.1136/heartasia-2014010494
}

\author{
ABSTRACT \\ Background Restoration of infarct vessel patency is the \\ key treatment for acute ST-elevation myocardial \\ infarction.
}

Objective The purpose of the study was to confirm the effectiveness of streptokinase (STK) for successful thrombolysis of the infarct-related artery (IRA) in patients with acute myocardial infarction (AMI), in relation to the time of the index event and age compared with newer thrombolytic agents, in a tertiary care centre.

Methods 100 patients (77\% male) thrombolysed with STK underwent coronary angiography within $48 \mathrm{~h}$ of presentation. Patency of the IRA was used to assess successful thrombolysis.

Results The mean pain-to-needle time was $3.24 \mathrm{~h}$. 76 patients $(76 \%)$ treated with thrombolysis had patent arteries with thrombolysis in myocardial infarction (TIMI) 2 or 3 flow. In subgroup analysis of time from the index event, patency rates were $83.3 \%, 77.5 \%, 68.7 \%$ and $40 \%$ in patients presenting within $0-2,2-4,4-6$ and 6-12 h, respectively. In subgroup analysis, all patients less than 30 years of age had patent arteries with TIMI 2 or 3 flow. Coronary angiography showed the IRA was the left anterior descending artery (LAD) in 55\%, the right coronary artery (RCA) in $33 \%$ and the left circumflex artery $(\mathrm{LCX})$ in $12 \%$. The patency rates of the LAD, RCA and LCX were $74.5 \%, 69.6 \%$ and $100 \%$, respectively.

Conclusions We found STK to be as effective as newer thrombolytic agents reported in other studies. In patients with AMl thrombolysed within $4 \mathrm{~h}$, STK results in higher patency in young compared to older patients.

\section{INTRODUCTION}

An occluded infarct-related artery (IRA) is associated with a doubling of long-term mortality. The benefits of thrombolytic therapy in patients with acute myocardial infarction (AMI) are well established, as shown by the meta-analyses of Yusuf et $a l^{1}$ and the Fibrinolytic Therapy Trialists (FTT) Collaborative Group, who reported that thrombolytic therapy decreases mortality at 35 days by $1.9 \% .^{2}$ Reperfusion is commonly assessed in terms of coronary blood flow, achievement of thrombolysis in myocardial infarction (TIMI) 3 flow being considered positive. The ischaemic time, door-to-balloon time and clinical risk are important determinants of favourable outcome and strong predictors of survival and preservation of left ventricular function. ${ }^{3}$ Longer delays from symptom onset to hospital presentation are associated with a reduced likelihood of receiving primary reperfusion therapy, and among those treated, late presenters had significantly longer door-to-balloon and door to-drug times.

In 1933 Tillet and Garner showed in laboratory tests that streptokinase (STK) obtained from the filtrate of group C $\beta$-haemolytic streptococcus could liquefy human fibrin. ${ }^{4}$ STK was initially used systemically, both experimentally and in patients, for venous and arterial thrombosis of recent onset. The results of extensive clinical trials with intravenous infusion of STK in acute myocardial infarction (MI), which were carried out in Europe and the USA during the late 1960 s and 1970 s, were inconclusive. The chief limitation of these studies was that treatment was initiated at variable times, starting between 4 and $6 \mathrm{~h}$ after the appearance of pain, and comparisons were based on mortality only as no angiographic control was available. However, studies on the effect of STK as demonstrated by angiography reported that the $90 \mathrm{~min}$ patency rate of STK was $50 \%$ while that of newer thrombolytics was $75 \% .^{5}$

This study was designed to assess the patency of the IRA in patients thrombolysed with STK in current era with newer antiplatelet and antithrombotic drugs.

\section{METHODS}

The study group consisted of 100 patients who were between 18 and 75 years of age and met the criteria for AMI for which revascularisation was indicated. These criteria were chest discomfort within the last $12 \mathrm{~h}$ in addition to one of the following: ST segment elevation of more than $2 \mathrm{~mm}$ in two or more contiguous precordial leads, ST segment elevation of more than $1 \mathrm{~mm}$ in two or more contiguous limb leads, posterior infarction (dominant R waves and ST depression in V1-V3), and new onset left bundle branch block. All patients were given the option of primary percutaneous transluminal coronary angioplasty (PTCA) or thrombolysis; those who refused primary PTCA were thrombolysed and enrolled in the study.

The exclusion criteria were: any prior intracranial haemorrhage, a known structural cerebral vascular lesion (eg, arteriovenous malformation), a known malignant intracranial neoplasm (primary or metastatic), ischaemic stroke within 3 months except for acute ischaemic stroke within $3 \mathrm{~h}$, suspected aortic dissection, active bleeding or bleeding diathesis (excluding menses), a significant closed head or facial trauma within the previous 3 months, severe uncontrolled hypertension (systolic blood pressure $>180 \mathrm{~mm} \mathrm{Hg}$ or diastolic blood pressure $>110 \mathrm{~mm} \mathrm{Hg}$ ). 
STK was administered at a dose of $1.5 \mathrm{MU}$ over $1 \mathrm{~h}$ according to institutional protocol. ${ }^{6}$ Rapid clearance of STK from the circulation occurs mainly via combination with plasma antibodies. Estimates of the elimination half-life vary considerably, with values of $18-37$ min reported in some studies, while others have demonstrated a half-life of up to $83 \mathrm{~min}$, particularly for the STK-plasminogen activator complex.

Successful thrombolysis was defined by clinical and ECG criteria. We used two criteria as markers of successful thrombolysis: (1) almost complete resolution of chest pain and (2) ECG findings indicating successful thrombolysis. The vertical height of ST segment elevation in the worst infarct lead with the maximum ST segment elevation, before and at $90 \mathrm{~min}$ after thrombolysis, was measured in mm. The ST segment was measured $80 \mathrm{~ms}$ from the J point, which corresponded to the peak ST elevation. The J point was defined as the beginning of the ST segment on ECG. The ECG criterion of successful thrombolysis was defined as $\geq 50 \%$ reduction in ST segment elevation after $90 \mathrm{~min}$ in the worst infarct lead with or without evidence of reperfusion arrhythmias.

ECG readings at baseline and $1.5 \mathrm{~h}$ after starting thrombolysis were obtained. Blood samples were collected at baseline and at 12 and $24 \mathrm{~h}$ after starting infusion in order to monitor serum levels of CK-MB, total CK and troponin. Each patient was followed up throughout their hospital stay, and 10\% underwent stenting for post-MI angina during hospitalisation.

In accordance with our protocol, angiography was performed within $48 \mathrm{~h}$ for all the patients, and patency rate according to TIMI was computed. Multivessel disease was defined as a reduction in lumen diameter of at least $50 \%$ in at least two major epicardial arteries. Reperfusion was judged to be successful if the IRA demonstrated TIMI grade 2 or 3 flow; TIMI grade 0 or 1 flow indicated no reperfusion. ${ }^{7}$

\section{Definitions of perfusion in the TIMI trial Grade 0 (no perfusion)}

In grade 0 , there is no antegrade flow beyond the point of occlusion. In grade 1 flow (penetration without perfusion), the contrast material passes beyond the area of obstruction but 'hangs up' and fails to opacify the entire coronary bed distal to the obstruction for the duration of the cineangiographic filming sequence.

\section{Grade 2 (partial perfusion)}

In grade 2 flow, the contrast material passes across the obstruction and opacifies the coronary bed distal to the obstruction. However, the rate of entry of contrast material into the vessel distal to the obstruction or its rate of clearance from the distal bed (or both) are perceptibly slower than its entry into or clearance from comparable areas not perfused by the previously occluded vessel-for example, the opposite coronary artery or the coronary bed proximal to the obstruction.

\section{Grade 3 (complete perfusion)}

In grade 3 flow, antegrade flow into the bed distal to the obstruction occurs as promptly as antegrade flow into the bed proximal to the obstruction, and clearance of contrast material from the involved bed is as rapid as clearance from an uninvolved bed in the same vessel or the opposite artery.

\section{Statistical analysis}

All the statistical analyses were carried out with the $\chi^{2}$ test and performed using the Statistical Package for Social Sciences V.16 (SPSS, Chicago, Illinois, USA).

\section{RESULTS}

Analysis of 100 patients who received STK during the study period and underwent coronary angiography was carried out. The mean age of the patients was $51.8 \pm 12.75$ years, and men accounted for $76.5 \%$ of the study population. The average body mass index was $26.61 \pm 3.65$. The patients' characteristics are given in table 1 . The mean pain-to-needle time was $3.24 \mathrm{~h}$.

Coronary angiography was performed within $48 \mathrm{~h}$ after the start of thrombolysis in all patients and TIMI flow grade 2 or 3 patency was achieved in $76 \%$ of patients receiving STK.

In subgroup analysis of time from the index event, those presenting within $0-2 \mathrm{~h}$ had a patency rate of $83.3 \%$, those presenting within $2-4 \mathrm{~h}$ a patency rate of $77.5 \%$, those presenting within $4-6 \mathrm{~h}$ a patency rate of $68.7 \%$, and those presenting after $6-12 \mathrm{~h}$ a patency rate of $40 \%$ (table 2). The TIMI flow grade 2 or 3 patency rate after STK correlated negatively with the time between the onset of pain and the beginning of thrombolysis (figure 1).

In the subgroup analysis of age with thrombolysis, we found that all patients less than 30 years of age with MI $(n=8)$ had patent arteries after STK. Patients aged 31-40, 41-50, 51-60 and $>60$ years had patency rates of $80 \%, 76.1 \%, 73.3 \%$ and $69.2 \%$, respectively (table 2). The TIMI flow grade 2 or 3 patency rate after STK correlated negatively with advancing age (figure 2).

In the subgroup analysis of patency of IRA in relation to STK, we found single-vessel disease in $53.96 \%$ and multivessel disease in $46.03 \%$ of patients receiving STK. In 55\% of these patients, the IRA was the left anterior descending artery (LAD), in $33 \%$ the IRA was the right coronary artery (RCA), and in $12 \%$ the IRA was the left circumflex artery (LCX). Of all patients presenting within $6 \mathrm{~h}, 74.5 \%$ of those with LAD disease had patent arteries, $69.6 \%$ of those with RCA disease had patent arteries and $100 \%$ of those with LCX disease had patent arteries (table 2, figure 3).

In the subgroup analysis of smoking, $87.5 \%$ of patients less than 30 years of age were smokers, while $80.0 \%, 80.9 \%, 56.6 \%$

Table 1 Baseline characteristics of study participants

\begin{tabular}{|c|c|}
\hline Baseline characteristics $(n=100)$ & N (\%) \\
\hline Age (years) & $51.8 \pm 12.75$ \\
\hline Male & 77 (77\%) \\
\hline History of smoking & $67(67 \%)$ \\
\hline Diabetes mellitus & $50(50 \%)$ \\
\hline Hypertension & $65(65 \%)$ \\
\hline Family history of coronary artery disease & $40(40 \%)$ \\
\hline Mean door-to-needle time (h) & 3.24 \\
\hline Anterior location of myocardial infarction & $55(55 \%)$ \\
\hline Successful thrombolysis & $76(76 \%)$ \\
\hline \multicolumn{2}{|l|}{ Antiplatelet drugs } \\
\hline Aspirin* & $100(100 \%)$ \\
\hline Clopidogrelt & $100(100 \%)$ \\
\hline Unfractionated heparin $\ddagger$ & $98(98 \%)$ \\
\hline$\beta$-Blockers & $88(88 \%)$ \\
\hline Nitrates & $90(90 \%)$ \\
\hline Statins & $80(80 \%)$ \\
\hline ACE inhibitors or angiotensin-receptor blockers & $56(56 \%)$ \\
\hline
\end{tabular}


Table 2 Study parameters and different variables regarding thrombolytic success

\begin{tabular}{llll}
\hline $\begin{array}{l}\text { Study } \\
\text { parameters }\end{array}$ & $\begin{array}{l}\text { No. of patients } \\
(\mathbf{n}=100)\end{array}$ & $\begin{array}{l}\text { No. of patients with } \\
\text { patent arteries } \\
(\mathbf{n}=76)\end{array}$ & $\begin{array}{l}\text { Successful } \\
\text { thrombolysis } \\
(\%)\end{array}$ \\
\hline $\begin{array}{l}\text { Age groups of study participants (years) } \\
20-30\end{array}$ & 8 & 8 & $100 \%$ \\
$31-40$ & 15 & 12 & $80 \%$ \\
$41-50$ & 21 & 16 & $76.1 \%$ \\
$51-60$ & 30 & 22 & $73.3 \%$ \\
$>60$ & 26 & 18 & $69.2 \%$ \\
Association between the index event and successful thrombolysis & \\
$0-2$ & 30 & 25 & $83.3 \%$ \\
$2-4$ & 49 & 38 & $77.5 \%$ \\
$4-6$ & 16 & 11 & $68.75 \%$ \\
$>6$ & 5 & 2 & $40.0 \%$ \\
Association between the IRA and successful thrombolysis & \\
LAD & 55 & 41 & $74.5 \%$ \\
RCA & 33 & 23 & $69.6 \%$ \\
LCX & 12 & 12 & $100 \%$ \\
\hline IRA, infarct-related artery; LAD, left anterior descending artery; LCX, left circumflex \\
artery; RCA, right coronary artery.
\end{tabular}

and $53.8 \%$, of those aged $31-40,41-50,51-60$ and $>60$ years, respectively, were smokers (table 3 ).

The 30-day outcome was available for only $80 \%$ of the study population. Overall, $12 \%$ of patients experienced major adverse cardiac events, defined as death, Q-wave MI and symptomdriven revascularisation (including in-hospital and 30-day data).

\section{DISCUSSION}

Intravenous fibrinolytic agents are routinely administered for acute re-establishment of vessel patency as large clinical trials have shown their unequivocal benefit. ${ }^{8}$

Our study showed that STK results in a patency rate of $76 \%$. This is in agreement with a study by Hogg et $a l^{9}$ where angiographic patency was assessed $90 \mathrm{~min}$ and $24 \mathrm{~h}$ after the start of therapy. Results showed that $55 \%$ of patients who received anistreplase and $53 \%$ of patients who received STK had patent IRAs (TIMI grade 2-3) at $90 \mathrm{~min}(95 \% \mathrm{CI} 42 \%$ to $68 \%$ and $95 \% \mathrm{CI}$ $40 \%$ to $66 \%$, respectively). At 24 h, $81 \%$ and $87.5 \%$ of arteries were patent, respectively (95\% CI $71 \%$ to $91 \%$ and $95 \%$ CI $83.5 \%$ to $91.5 \%$ ).

Our study is in disagreement with a few other studies. The TIMI phase one trial compared the effectiveness of STK to rt-PA in opening occluded arteries, and demonstrated that t-PA was superior to STK and had twice the rate of open arteries at $90 \mathrm{~min}(70 \%$ vs $43 \%)$ independent of the baseline angiogram. ${ }^{7}$

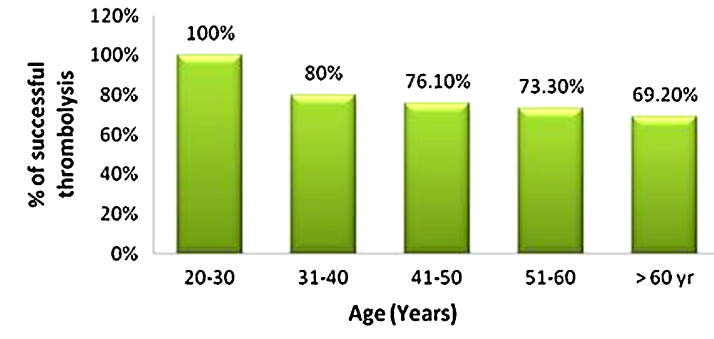

Figure 2 Association between age and thrombolysis.

The European Cooperative Study Group reported similar results. $^{10}$

In a study by Meyer et al, ${ }^{11}$ patency rates at $60 \mathrm{~min}$ were $71.8 \%$ for recombinant single-chain urokinase plasminogen activator (rscu-PA) and $48.0 \%$ for STK $(\mathrm{p}<0.001)$, while at $90 \mathrm{~min}$ they were $71.2 \%$ and $63.9 \%$, respectively $(p=0.15)$. At $24-$ $36 \mathrm{~h}, 6 / 121$ patients treated with rscu-PA and 5/114 patients treated with STK showed reocclusion of the vessel. Thus intravenous administration of rscu-PA resulted in a higher patency rate, earlier reperfusion, less disturbance of haemostasis, and fewer bleeding complications than intravenous administration of STK.

The higher patency rates found in our study may be because patients with AMI arrive relatively early at our hospital due to its central location and therefore generally derive maximum benefit from thrombolytic therapy. While, ideally, patients should receive STK as soon as possible after symptom onset, late benefit has been observed in patients presenting up to $12 \mathrm{~h}$ after pain onset, as is often the case with the elderly.

Although $90 \mathrm{~min}$ patency is considered optimal in other studies, we planned to perform angiograms within $48 \mathrm{~h}$ rather than at $90 \mathrm{~min}$ because the high thrombus load at $90 \mathrm{~min}$ and patient were not to be taken for facilitated percutaneous coronary intervention.

In subgroup analysis of time from the index event, those presenting earlier after the index event had a higher patency rate compared to those presenting later.

The patency rate after STK administration correlated negatively with the time between the onset of pain and the beginning of thrombolysis. The results of this analysis suggest that the efficacy of STK in restoring early coronary patency after intravenous thrombolysis is markedly reduced when patients are treated more than 3-4 h after pain onset. This confirms a preliminary observation in the TIMI- 1 study and PERM study. ${ }^{12}$

In the subgroup analysis of age with thrombolysis, we found that all patients less than 30 years of age with MI, had patent arteries after STK. To the best of our knowledge, this interesting association has not previously been reported in the literature.

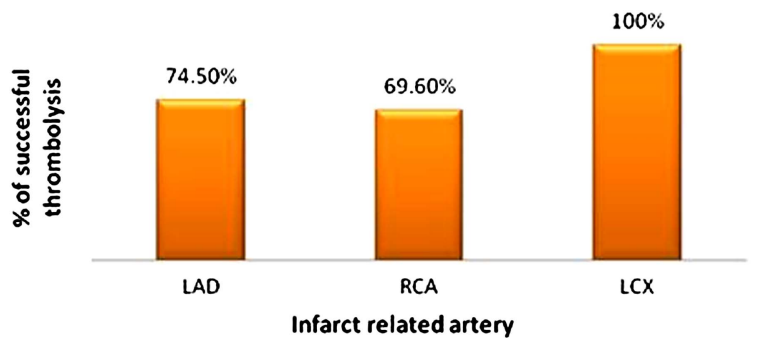

Figure 3 Association between infarct-related artery and thrombolysis. $L A D$, left anterior descending artery; LCX, left circumflex artery; RCA, right coronary artery.

Figure 1 Association between the index event and thrombolysis. 
Table 3 Smokers in the study population

\begin{tabular}{lccl}
\hline Age group (years) & No. of patients & No. of smokers & Percentage \\
\hline $20-30$ & 8 & 7 & $87.5 \%$ \\
$31-40$ & 15 & 12 & $80.0 \%$ \\
$41-50$ & 21 & 17 & $80.9 \%$ \\
$51-60$ & 30 & 17 & $56.6 \%$ \\
$>60$ & 26 & 14 & $53.8 \%$ \\
& 100 & 67 & $67 \%$ \\
\hline
\end{tabular}

The TIMI flow grade 2 or 3 patency rate after STK correlated negatively with advancing age.

In the subgroup analysis, we found that the LCX had a higher patency rate. This might be because the LCX has a smaller diameter than the LAD or RCA and hence a smaller thrombus burden. Data on the influence of infarct location on arterial patency rates after thrombolytic therapy have been conflicting. Some investigators have reported a higher reperfusion rate after STK infusion in the LAD than in other arteries, ${ }^{13}$ others have reported similar reperfusion rates in the LAD and RCA with lower rates in the LCX, ${ }^{14}{ }^{15}$ while one registry reported equivalent patency rates in all three arteries. ${ }^{16}$

In the subgroup analysis of smokers, $87.5 \%$ of patients less than 30 years of age were smokers, while the majority $(80 \%)$ of patients between 30 and 50 years of age were also smokers.

The proportion of patients with patency of the IRA did not differ with respect to the site (proximal, mid or distal) of the coronary occlusion. Gender did not affect the incidence of reperfusion.

\section{Study limitations}

This study had several limitations. The sample size was small, STK was not compared with newer thrombolytic agents and angiographic patency has been shown to be poorly reproducible, with a notable overlap between patients with TIMI flow grades 2 and 3.

\section{CONCLUSION}

In our study we found STK to be as effective as the newer thrombolytics reported in other studies. This drug is especially important in countries like India, where relatively young patients experience MI. In addition, primary angioplasty is carried out at few centres in India and so it is better to thrombolyse young patients soon after MI and carry out angiography or stress tests later, as indicated. STK remains a useful treatment in developing countries and large scale studies are required to show that it is still effective.
Contributors RKG performed the procedures, provided the concept for the manuscript, and analysed and interpreted the data. SG performed the review, acquired the data and wrote the manuscript. DSB and DP were responsible for the literature search, statistical analysis and editing of the manuscript.

\section{Competing interests None.}

Ethics approval The Ethics Committee of JLN Medical College, Ajmer, Rajasthan, India approved the study.

Provenance and peer review Not commissioned; externally peer reviewed.

\section{REFERENCES}

1 Yusuf S, Collins R, Peto R, et al. Intravenous and intracoronary fibrinolytic therapy in acute myocardial infarction: overview of results on mortality, reinfarction and side-effects from 33 randomized controlled trials. Eur Heart J 1985:6:556-85.

2 Fibrinolytic Therapy Trialists' (FTT) Collaborative Group. Indications for fibrinolytic therapy in suspected acute myocardial infarction: collaborative overview of early mortality and major morbidity results from all randomized trials of more than 1000 patients. Lancet 1994:343:311-22.

3 Lachance P, Déry JP, Beaudoin J, et al. ECG-guided immediate intervention at the time of primary $\mathrm{PCI}$ to reduce door-to-balloon time in ST-elevation myocardial infarction patients. J Invasive Cardiol 2008;20:623-6.

4 Tillet WS, Garner RL. The fibrinolytic activity of hemolytic streptococci. J Exp Med 1933;58:485.

5 Antman EM, Morrow DA. ST-Segment Elevation Myocardial Infarction: Management. In: Bonow RO, Mann DL, Zipes DP, Libby P, eds. Braunwald's Heart Disease: A Textbook of Cardiovascular Medicine. 9th ed. Philadelphia: Saunders Elsevier, 2011:1124.

6 Topol EJ, Ohman EM, Armstrong PW, et al. Survival outcomes 1 year after reperfusion therapy with either alteplase or reteplase for acute myocardial infarction: results from the Global Utilization of Streptokinase and t-PA for Occluded Coronary Arteries (GUSTO) III Trial. Circulation 2000;102:1761-5.

7 The TIMI Study Group. The Thrombolysis In Myocardial Infarction (TIMI) trial, phase 1. N Eng J Med 1985;312:932-6.

8 Centurión OA. The open artery hypothesis: beneficial effects and long-term prognostic importance of patency of the infarct-related coronary artery. Angiology 2007;58:34-44

9 Hogg KJ, Gemmill JD, Burns JMA, et al. Angiographic patency study of anistreplase versus streptokinase in acute myocardial infarction. Lancet 1990;335:254-8.

10 Verstraete $M$, Bernard $R$, Bory $M$, et al. Randomised trial of intravenous recombinant tissue-type plasminogen activator versus intravenous streptokinase in acute myocardial infarction. Report from the European Cooperative Study Group for Recombinant Tissue-type Plasminogen Activator. Lancet 1985;1:842-7.

11 Meyer J, Bar F, Barth $\mathrm{H}$, et al. Randomised double-blind trial of recombinant pro-urokinase against streptokinase in acute myocardial infarction: PRIMI Trial Study Group. Lancet 1989:333:863-8.

12 Steg PG, Laperche T, Golmard JL, et al. Efficacy of streptokinase, but not tissue-type plasminogen activator, in achieving 90-minute patency after thrombolysis for acute myocardial infarction decreases with time to treatment. J Am Coll Cardiol 1998:31:776-9.

13 Tendera MP, Campbell WB, Tennant SN, et al. Factors influencing probability of reperfusion with intracoronary ostial infusion of thrombolytic agent in patients with acute myocardial infarction. Circulation 1985;71:124-8.

14 Kennedy JW, Genjini GG, Timmis GC, et al. Acute myocardial infarction treated with intracoronary streptokinase: a report of the Society for Cardiac Angiography. Am J Cardiol 1985;55:X71-7

15 Merx W, Dorr R, Rentrop KP, et al. Evaluation of the effectiveness of intracoronary streptokinase infusion in acute myocardial infarction: postprocedure management and hospital course in 104 patients. Am Heart J 1981;102:1131-7.

16 Weinstein J. The international registry to support approval of intracoronary streptokinase thrombolysis in the treatment of myocardial infarction: assessment of safety and efficacy. Circulation 1983;68(2 Pt 2):161-6. 